\title{
Design Methodology and Performance Evaluation of a Tapered Cell
}

\author{
Wen-Tron Shay, Member, IEEE, Wei-Ping Hong, Ray-Rong Lao, and Jenn-Hwan Tarng, Senior Member, IEEE
}

\begin{abstract}
Tapered cells are used in the calibration of electric field (E-field) probes. However, the design methodology of tapered cells has not been publicly reported in detail. This paper introduces the structure of a tapered cell and proposes a theoretical model for characteristic impedance calculation. Based on this model and developed design rules, an impedance-matched tapered cell was constructed. The measured characteristic impedances and impedance distribution along the cell show favorable agreement with the design goals, verifying the accuracy of the design methodology. The field distributions were also measured. The results prove that the cell is useful for calibrating E-field probes.
\end{abstract}

Index Terms-Calibration, electric fields (E-fields), impedance matching, measurement, transmission lines.

\section{INTRODUCTION}

$\mathbf{E}$ LECTRIC field (E-field) probes are widely used in electromagnetic compatibility (EMC) radiated immunity tests [1] and in environmental electromagnetic field strength measurements [2]. To ensure their accuracy, the probes must be periodically calibrated. There are two main calibration methods. One method is calibrating the probe under test with standard E-fields, the field strengths of which are measured and calibrated using a transfer standard (such as a precision E-field probe) traceable to national standards. The other method is calibrating the probe under test using standard E-fields with calculated field strengths [3]. For either method, a facility is required to generate standard E-fields for calibrations. Several facilities are used for generating the required standard E-fields. Among them, TEM cells and anechoic chambers are the most common facilities. For frequencies lower than a few hundred megahertz, TEM cells are preferred because they need less cost/space and are easier to operate. In addition, the TEM cell is fully shielded, and most electromagnetic (EM) energy is constrained in the cell. High-level E-fields can be generated in the cell with minimal input power, and no measure is required to avoid interfering with nearby equipment. A TEM cell consists

Manuscript received July 3, 2012; revised November 4, 2012; accepted December 31, 2012. Date of publication March 7, 2013; date of current version May 8, 2013. The Associate Editor coordinating the review process for this paper was T. Lipe.

W.-T. Shay is with the Center for Measurement Standards, Industrial Technology Research Institute, Hsinchu 300, Taiwan, and also with the Institute of Communications Engineering, National Chiao Tung University, Hsinchu 300, Taiwan (e-mail: wtshay@itri.org.tw).

W.-P. Hong and R.-R. Lao are with the Center for Measurement Standards, Industrial Technology Research Institute, Hsinchu 300, Taiwan (e-mail: hongweiping@itri.org.tw; rrl@itri.org.tw).

J.-H. Tarng is with the Institute of Communications Engineering, National Chiao Tung University, Hsinchu 300, Taiwan (e-mail: j4t@mail.nctu.edu.tw).

Digital Object Identifier 10.1109/TIM.2013.2241512 of a section of expanded rectangular coaxial line (main section) and two tapered transition sections (to adapt coaxial connectors) at each end of the cell. The main section and the tapered sections are designed to have a characteristic impedance of $50 \Omega$ along the cell to insure minimum reflections. The main section consists of a rectangular outer conductor and a flat center septum. The probe under test is typically placed at the center of the upper or lower part of the main section during calibration. If the maximum probe dimension is less than one third of the separation between the top (or lower) wall and the center septum, the uncertainty of the field strength less than $1 \mathrm{~dB}$ can be obtained [4]. The maximum operating frequency of a TEM cell is limited by the cutoff frequencies of the higher order modes, which are determined by the cell dimensions. For larger cells, the cutoff frequencies are lower. To be used at higher frequencies, the TEM cell must be small, and consequently, the size of the uniform test volume is small. Small TEM cells are only applicable to small devices, such as specific absorption rate (SAR) probes. Therefore, for frequencies higher than a few hundred megahertz, anechoic chambers with standard transmitting antennas are commonly used to generate uniform standard fields. At a point on the axis (boresight) of the transmitting antenna, the E-field strength can be accurately calculated with the distance from the transmitting antenna to the point, the net input power to the transmitting antenna, and the gain of the transmitting antenna. With a series of standard antennas (such as rectangular pyramidal horns), the usable frequency range from $450 \mathrm{MHz}$ to $40 \mathrm{GHz}$ is reported [3]. The measurement uncertainty less than $1 \mathrm{~dB}$ can be also obtained with an anechoic chamber calibration system [5]. The anechoic chamber is large; therefore, it can provide a large test volume. However, it requires a large amount of space due to its size. The construction and the subsequent maintenance of an anechoic chamber are also expensive. In addition, expensive highpower RF amplifiers are usually required to generate high-level E-fields. Comparing the two facilities, TEM cells are relatively inexpensive but only suitable for lower frequencies; anechoic chambers provide calibrations over broadband frequencies and have a larger test volume, but are much more expensive.

Another standard field-generating facility is the gigahertz transverse electromagnetic (GTEM) cell. Basically, the GTEM cell is a tapered asymmetrical rectangular coaxial transmission line terminated with resistors and absorbers. The GTEM cell is much larger than TEM cells and can provide a larger test volume. The usable frequency range of the GTEM cell is from $9 \mathrm{kHz}$ to $1 \mathrm{GHz}$. Generally, the calibration method with transfer standard is applied to reduce the measurement uncertainty. Measurement uncertainty of less than $1 \mathrm{~dB}$ is reported [6]. 

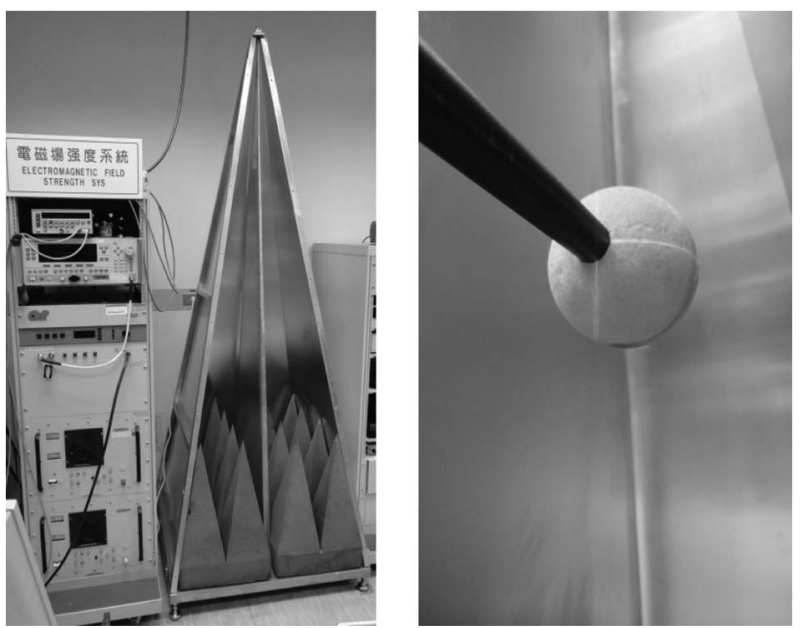

Fig. 1. (Left) Tapered cell and (right) probe calibration applying laser alignment.

However, the GTEM cell is also large in size and much more expensive than TEM cells. Furthermore, the frequency range cannot cover all frequencies for mobile communications, which significantly reduces it availability.

In contrast, tapered cells can be used as field generators over the most commonly used frequencies, including the bands used for mobile communications [7]. In addition, tapered cells also have the advantages that TEM cells have, including a lower construction cost (comparable to TEM cells), a smaller space requirement, and less required RF power. In our experience, calibrating E-field probes with a tapered cell is rather convenient, and the measurement uncertainty (by using a transfer standard) of less than $1 \mathrm{~dB}$ is also achievable.

To our knowledge, the tapered cell was invented by the National Physical Laboratory (NPL, U.K.), and one tapered cell has been used as calibration equipment in NPL for many years [8]. However, the design methodology of tapered cells has not been publicly reported in detail. This paper first introduces the structure of a tapered cell and then provides a theoretical model for characteristic impedance calculation. In addition, design rules to obtain constant characteristic impedance along the cell are described. Based on the proposed model and design rules, this study constructed a tapered cell with a usable frequency range from $200 \mathrm{MHz}$ to $2.5 \mathrm{GHz}$. Three center septa with differing dimensions were fabricated to provide various cell impedances for verifying the theoretical model. The characteristic impedances at six cross sections and the distribution along the cell were measured using a time-domain reflectometer (TDR). The results show favorable agreement with the calculated results. E-field distributions were also investigated with two orthogonal dipole sensors.

\section{Structure of TAPered Cells}

The tapered cell constructed in our laboratory is shown in Fig. 1 (left panel). On the top of the cell, an adaptor is used to adapt the coaxial connector to the rectangular cell body. The cell body of the tapered cell is similar to the tapered section of TEM cells. However, one side of the outer conductor is removed to allow the placement of the probe under test. The

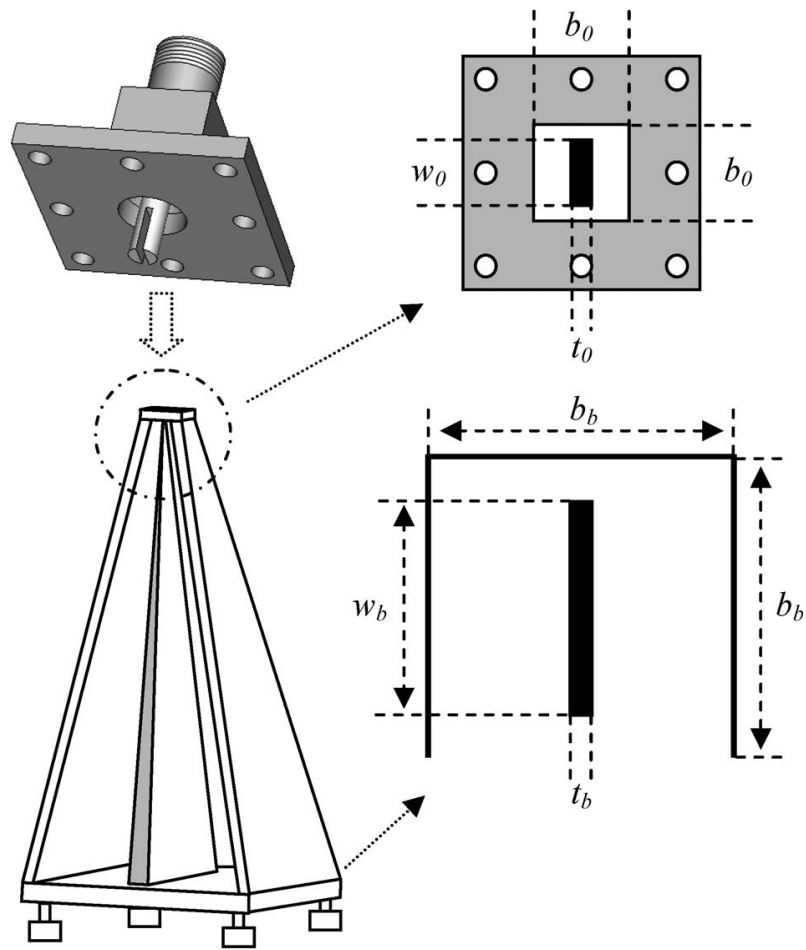

Fig. 2. Detailed structure of the tapered cell. (Upper left) Adaptor. (Upper right) Cross-sectional view of the top. (Lower left) Cell body. (Lower right) Cross-sectional view of the bottom.

end of the center septum is electrically connected to the bottom ground plane (outer conductor). Absorbers are placed on the bottom to reduce the reflection from the bottom. The performance of the absorbers may influence the usable frequency range. Tapered cells radiate RF energy to their surroundings, which can be hazardous to operators and may cause interference to nearby instruments. To prevent this, in our laboratory, two movable racks mounted with absorbers are placed in front of the open side of the cell during operation.

A probe calibration applying laser alignment is shown in Fig. 1 (right panel). Easier probe positioning is also an advantage of tapered cells. Fig. 2 shows the metal structure of the cell in detail. The cell body consists of an outer conductor (three metal walls and bottom) and a center septum. Four adjustable supports are used to level the cell. The adaptor is a type-N connector followed by an expanded transition section and can be screwed to the cell body. The extended part of the center pin is slotted to allow insertion of the center septum. The output port of the adaptor is coaxial; however, the input port of the cell body is rectangular. Therefore, reflection at the joint plane is nearly unavoidable, although this can be reduced to an acceptable level through careful design.

To ensure easier design and fabrication, the cross section of the cell is square, and the center septum is fixed at the center to make the cell symmetric. The key dimensional parameters in the fabrication process are the dimensions at the top and bottom of the cell: $b_{0}, w_{0}, t_{0}, b_{b}, w_{b}$, and $t_{b}$. The expanding angles shown in Fig. 3 determine the dimensions of the cell and the characteristic impedance distribution along the cell. Section III discusses how to obtain constant impedance by selecting specific angles. 


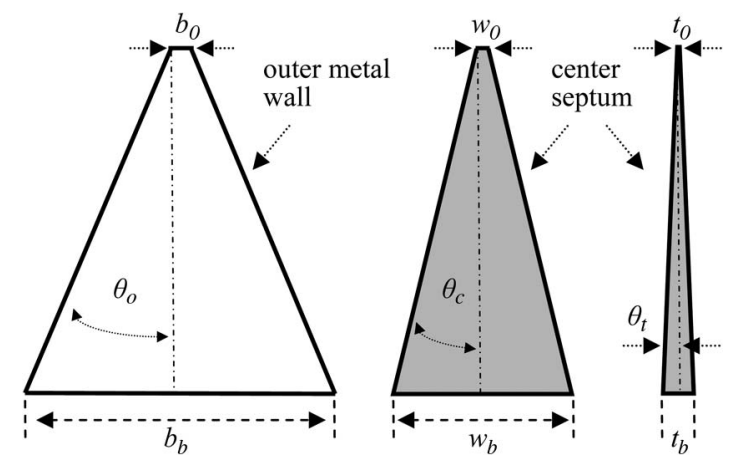

Fig. 3. Expanding angles of the tapered cell. (Left) Inner expanding angle of the outer metal wall. (Center) Expanding angle of the center septum in width. (Right) Expanding angle of the center septum in thickness.

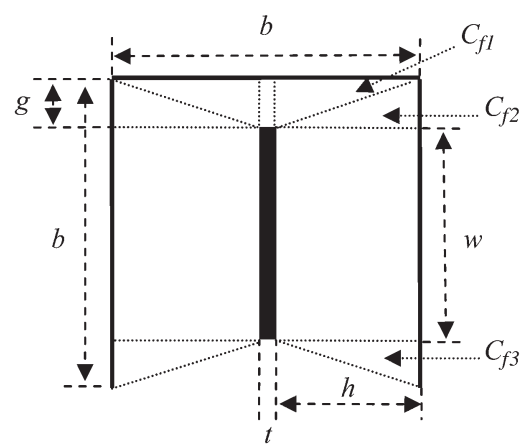

Fig. 4. Cross-sectional view of the tapered cell and its capacitance distribution.

\section{Characteristic IMPedAnCE MOdel}

For a lossless uniform two-wire transmission line, the characteristic impedance of this type of line for TEM waves is given by [9], [10]

$$
Z_{c}=\sqrt{\mu_{0} \varepsilon_{0}} / C
$$

where $\mu_{0}$ and $\varepsilon_{0}$ are the free-space permeability and permittivity, respectively, and $C$ is the capacitance per unit length of the transmission line $(\mathrm{F} / \mathrm{m})$. The characteristic impedance of the line is mainly controlled by $C$ because $\mu_{0}$ and $\varepsilon_{0}$ are constant. Although the body section of the tapered cell is not uniform, it can be considered as a cascade of very short (infinitesimal) uniform two-wire transmission lines. Each short uniform line can use (1) to design its characteristic impedance. If the dimensions of every short uniform line are chosen to have a characteristic impedance of $50 \Omega$, the reflections due to the mismatch between adjacent short uniform lines can be minimized. In other words, if the characteristic impedance of every cross section of a tapered cell is designed to be $50 \Omega$ by using (1), then the reflections in the cell can be minimized. This is one of the design goals of this study.

Fig. 4 shows an arbitrary cross-sectional view of the tapered cell. To the best of our knowledge, no model is available for calculating the characteristic impedance of this type of structure. If the structure is divided into upper and lower sections, it is observed that the upper section is a part of a rectangular line and the lower section is a part of a shielded strip transmission line, respectively. Thus, to simplify analysis, we properly divided the capacitance between the outer and center conductors into nine partial capacitances [11], as shown in Fig. 4. The total capacitance $C$ can be written as the sum of all partial capacitances, i.e.,

$$
C=\varepsilon_{0}\left(\frac{2 w}{h}+\frac{t}{g}\right)+2\left(C_{f 1}+C_{f 2}+C_{f 3}\right) .
$$

Fringe capacitances $C_{f 1}$ and $C_{f 2}$ can be obtained from a rectangular line model [10], i.e.,

$$
\begin{aligned}
& C_{f 1}=\frac{\varepsilon_{0}}{\pi}\left[\log \left(\frac{g^{2}+h^{2}}{4 h^{2}}\right)+2\left(\frac{h}{g}\right) \arctan \left(\frac{g}{h}\right)\right] \\
& C_{f 2}=\frac{\varepsilon_{0}}{\pi}\left[\log \left(\frac{g^{2}+h^{2}}{4 g^{2}}\right)+2\left(\frac{g}{h}\right) \arctan \left(\frac{h}{g}\right)\right]
\end{aligned}
$$

whereas fringe capacitance $C_{f 3}$ can be calculated using a shielded strip line formula [12], i.e.,

$$
\begin{aligned}
C_{f 3}=\frac{\varepsilon_{0}}{\pi}[ & \frac{2}{1-t / b} \ln \left(\frac{1}{1-t / b}+1\right) \\
& \left.\quad-\left(\frac{1}{1-t / b}-1\right) \ln \left(\frac{1}{(1-t / b)^{2}}-1\right)\right] .
\end{aligned}
$$

The usable condition of (5) is when $w /(b-t)>=0.35$. This is because as the width of the center septum $(w)$ decreases, the fringe fields on both sides of a shielded strip line interact with each other, causing the formula to become inaccurate. However, this should not pose a problem for tapered cells because wide center septa are typically designed to have a large uniform test volume.

\section{Constant Characteristic IMPedance Design}

Using (1)-(5) from Section III (hereafter referred to as the impedance model), we can calculate the characteristic impedance of any selected position (cross section). However, as the tapered cell is expanded, the dimensions vary along the vertical direction. Therefore, if a cell is not properly designed, the characteristic impedance will vary at different positions.

Referring to (1)-(5), we can find that the capacitances depend on the ratios $t / b, g / h, t / g$, and $w / h$. Therefore, if the ratios are kept constant, then the capacitances and the characteristic impedance will be constant along the cell. To achieve this result, every cross-sectional dimension has to be proportionally expanded, including the thickness $(t)$ of the center septum. This differs from TEM cells. The thickness of the center septum of a TEM cell is typically constant. This point can be illustrated by comparing the characteristic impedance variations when the center septum is and is not proportionally expanded, as shown in Fig. 5. The original dimensions of the cross section are set to be $b=4 \mathrm{~cm}, w=3.5 \mathrm{~cm}$, and $t=0.2 \mathrm{~cm}$, and are multiplied by figures ranging from 1 to 20 in order to provide different characteristic impedances for comparison. Calculations based on the impedance model and software simulations (with CST Microwave Studio) are both applied. The results show that the characteristic impedances increase when $t$ is constant and nearly keep constant when $t$ is proportionally expanded. For $t$ _constant curves, when the dimensions are large, $t$ is no longer critical; the characteristic impedance is therefore gradually approaching a fixed value. The maximum difference between

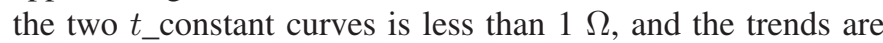
nearly identical. For $t_{-}$expanded curves, the calculation and 


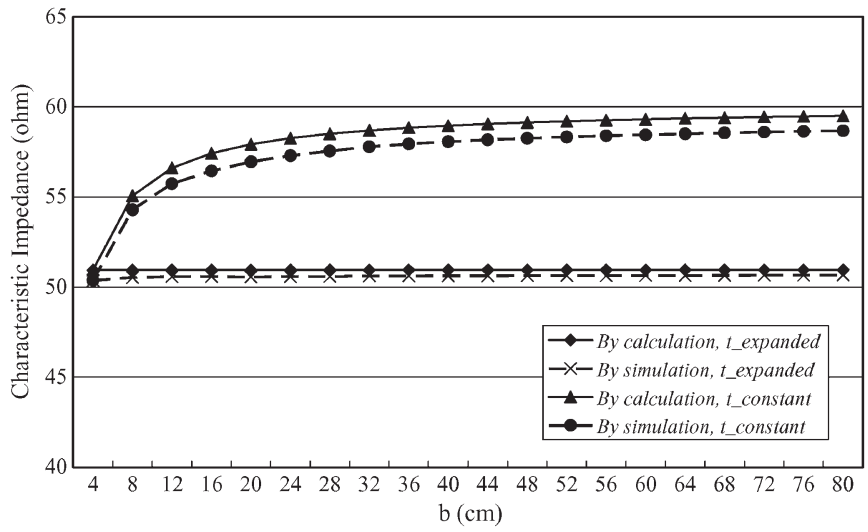

Fig. 5. Comparison of the characteristic impedances obtained by calculation and simulation.

simulation results are close to each other and nearly constant as the dimensions expanded. These results also verify the accuracy of the impedance model. Although modern simulation software products can be used in tapered cell design (as we did in the beginning of this study), the impedance model is more effective both in designing and in diagnosing tapered cells. The simulation was used only as a verification tool in this study.

In designing the tapered cell, we first determined the dimensions of the outer conductor and then focused on designing the center septum. For the outer conductor, the height was $236 \mathrm{~cm}$ $\left(c_{h}\right)$, and the outer and inner width (excluding the thickness of the outer conductor) at the bottom were 91 and $86 \mathrm{~cm}$ $\left(b_{b}\right)$, respectively. Larger cells may have a larger uniform test volume; however, in this study, cell size was limited because of the space available in our laboratory. The width $\left(b_{0}\right)$ of the top opening (see Fig. 2) was $1.5 \mathrm{~cm}$ in our design and was determined by considering how well it matched the adaptor. The expanding angle $\theta_{0}$ was then calculated as

$$
\theta_{0}=\tan ^{-1}\left(\left(b_{b}-b_{0}\right) / 2 c_{h}\right)=10.15^{\circ} .
$$

After the dimensions of the outer conductor were known, we determined the top dimensions $w_{0}$ and $t_{0}$ of the center septum with the impedance model. However, there are infinite pairs of $w_{0}$ and $t_{0}$ that can obtain a characteristic impedance of $50 \Omega$. A larger $w_{0}$ is typically preferred because the uniform test volume is larger. However, if the values of $w_{0}$ and $b_{0}$ are close, small mechanical deviations might cause significant impedance changes. In our design, $w_{0}$ of $1.3 \mathrm{~cm}$ was chosen, and thereafter, $t_{0}$ was calculated to be $0.09 \mathrm{~cm}$.

As mentioned, the dimensional ratios must remain constant to obtain constant characteristic impedance along the cell. There are two methods to achieve this result. The first is to derive a pair of specific expanding angles $\theta_{t}$ and $\theta_{c}$ that can keep the dimensional ratios constant along the cell. Thereafter, the dimensions of the bottom can be calculated. The second method is the reverse of the first method because it calculates the dimensions of the bottom by applying the ratios of the top and then determines the expanding angles. The first method is described here. The ratio $t / b$ is considered first. At any distance $(d)$ from the top, the ratio must be equal to $t_{0} / b_{0}$, that is

$$
\frac{t}{b}=\frac{t_{0}+2 d \tan \theta_{t}}{b_{0}+2 d \tan \theta_{0}}=\frac{t_{0}}{b_{0}} .
$$

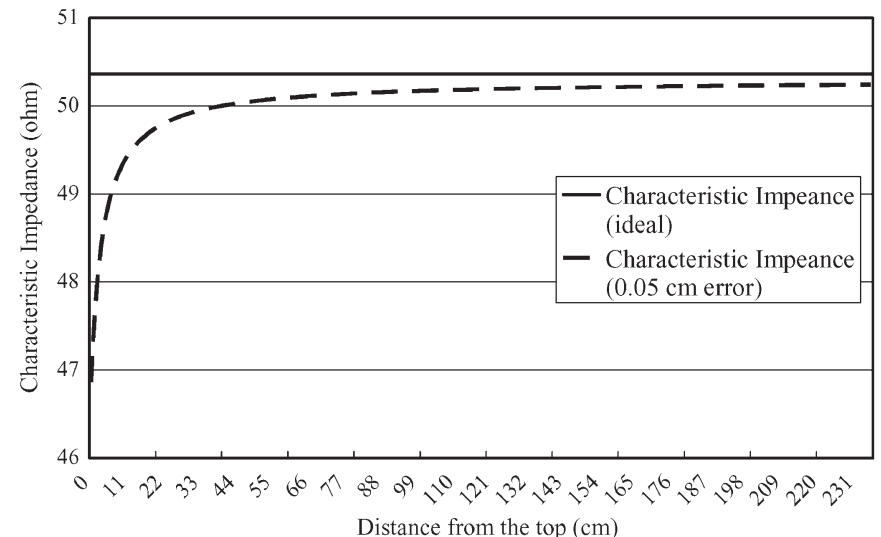

Fig. 6. Calculated characteristic impedances along the cell.

Following some mathematical manipulations, we have

$$
\theta_{t}=\tan ^{-1}\left(\frac{t_{0} \tan \theta_{0}}{b_{0}}\right) \text {. }
$$

For $w / h$, at any distance $d$, the ratio can be expressed as

$$
\frac{w}{h}=\frac{w_{0}+2 d \tan \theta_{c}}{\left(b_{0}+2 d \tan \theta_{0}-t_{0}-2 d \tan \theta_{t}\right) / 2}=\frac{w_{0}}{\left(b_{0}-t_{0}\right) / 2} .
$$

Thereafter, the expanding ratio $\theta_{c}$ can be derived as

$$
\theta_{c}=\tan ^{-1}\left[\frac{w_{0}\left(\tan \theta_{0}-\tan \theta_{t}\right)}{\left(b_{0}-t_{0}\right)}\right] .
$$

If $t / b$ and $w / h$ are constant, then $g / h$ and $t / g$ must be also constant.

The calculated $\theta_{t}$ and $\theta_{c}$ were $0.62^{\circ}$ and $8.82^{\circ}$, respectively. Then, the results $w_{b}=74.5 \mathrm{~cm}$ and $t_{b}=5.1 \mathrm{~cm}$ were calculated. With the dimensions obtained above, we are able to fabricate a tapered cell with constant characteristic impedance. This study fabricated three center septa for verifying the impedance model. The above derived dimensions and angles were for Septum No. 3.

The solid line in Fig. 6 shows the calculated results with the dimensions of Septum No. 3. The characteristic impedance is constant along the cell, as expected. Tapered cells are relatively large. The fabrication of the cells without any dimensional deviation is difficult. In addition, even stress or gravity can slightly alter the cell dimensions. The dotted line in Fig. 6 demonstrates how mechanical tolerance influences the distributed impedance. Dimensions are the same as for the solid line, except $w_{0}$. The top width $w_{0}$ is set to be $1.35 \mathrm{~cm}$, allowing a $0.05-\mathrm{cm}$ deviation. We then find that the characteristic impedances undergo notable changes near the top because the deviation is comparable at this section. The influence becomes gradually insignificant as the distance increases.

\section{MOdEL VERIFICATION AND PERFORMANCE EVALUATION}

A tapered cell with the dimensions obtained in Section IV was constructed, as shown in Fig. 1. Characteristic impedances and field distributions were measured and analyzed as follows. 
TABLE I

VERIFICATION OF THE IMPEDANCE MODEL

\begin{tabular}{|c|c|c|c|c|c|c|}
\hline $\begin{array}{c}\text { Septum } \\
\text { No. }\end{array}$ & $\begin{array}{c}t \\
(\mathrm{~cm})\end{array}$ & $\begin{array}{c}g \\
(\mathrm{~cm})\end{array}$ & $\begin{array}{c}b \\
(\mathrm{~cm})\end{array}$ & $\begin{array}{c}w \\
(\mathrm{~cm})\end{array}$ & $\begin{array}{c}\text { Measured } \\
(\Omega)\end{array}$ & $\begin{array}{c}\text { Calculated } \\
(\Omega)\end{array}$ \\
\hline \multirow{2}{*}{1} & 1.5 & 4.5 & 39.5 & 30.0 & $60.6 \pm 0.9$ & $60.7 \pm 0.3$ \\
\cline { 2 - 7 } & 1.2 & 4.0 & 34.2 & 26.2 & $60.7 \pm 1.0$ & $60.9 \pm 0.3$ \\
\hline \multirow{2}{*}{2} & 2.6 & 4.1 & 47.4 & 39.9 & $53.3 \pm 0.7$ & $53.7 \pm 0.3$ \\
\cline { 2 - 7 } & 3.5 & 5.9 & 65.3 & 56.2 & $53.4 \pm 0.5$ & $53.5 \pm 0.3$ \\
\hline \multirow{2}{*}{3} & 3.1 & 3.7 & 49.2 & 42.8 & $50.4 \pm 0.6$ & $50.7 \pm 0.3$ \\
\cline { 2 - 7 } & 3.4 & 4.2 & 53.9 & 46.8 & $50.5 \pm 0.6$ & $51.0 \pm 0.3$ \\
\hline
\end{tabular}

\section{A. Characteristic Impedance}

Three center septa were fabricated for verifying the impedance model. Replacing the center septum created differing characteristic impedances for the tests. Two arbitrary cross sections were selected when each center septum was configured in the cell.

Measured cross-sectional dimensions were used in the calculations with the impedance model. The measurement uncertainties of the dimensions will cause variations of the calculated results. In this paper, the distribution of the calculated characteristic impedances was obtained by thousands of calculations. For each calculation, the parameters $(t, b, g, w)$ were randomly varied in their possible dimensional range. Two times the standard deviation of the distribution was taken as the estimated calculation uncertainty.

A TDR was used in the characteristic impedance measurement. During the measurement, a precision reference air line $(50 \pm$ $0.1 \Omega$ ) and a short coaxial cable (just for connection) were connected between the TDR and the tapered cell. The reference air line was used as a standard to calibrate the TDR. Consequently, the measurement uncertainty was significantly reduced. The evaluated expanded uncertainty (coverage factor $k=2$ ) of the measurement is $0.3 \Omega$. The measured and calculated results and their corresponding uncertainties are listed in Table I. The differences between the measured and calculated results are rather small. The results confirm the accuracy of the impedance model.

In addition, the characteristic impedance distribution along the cell (starting at the mating surface of the adaptor) was measured with the TDR, and the result is shown in Fig. 7. Center Septum No. 3 was used in the measurement. The characteristic impedance was designed to be $50.4 \Omega$, which is slightly higher than $50 \Omega$ to allow for a possible loading effect when inserting the probe under test into the cell [4]. The impedances at the adaptor and the section near the top have obvious deviations from the design goal; the causes have been previously explained. However, the values are still good enough for matching. The average characteristic impedance in the $60-235-\mathrm{cm}$ range is $50.8 \Omega$, which is very close to the designed value. Furthermore, the maximum impedance variation in the range is only $0.7 \Omega$. These results prove that the design rules are useful.

The reflection coefficients of the cell were measured with an Agilent E5071C vector network analyzer (VNA) calibrated with a one-port calibration technique. The measurement reference plane was at the mating surface of the adaptor. The reflections from the adaptor, cell body, and the absorbers all contribute to the measurement results shown in Fig. 8. For lower frequencies, reflections are strong because the absorbers are ineffective for this band. If the acceptable criterion is $\mathrm{S} 11<-10 \mathrm{~dB}$, the usable frequency range is from $150 \mathrm{MHz}$

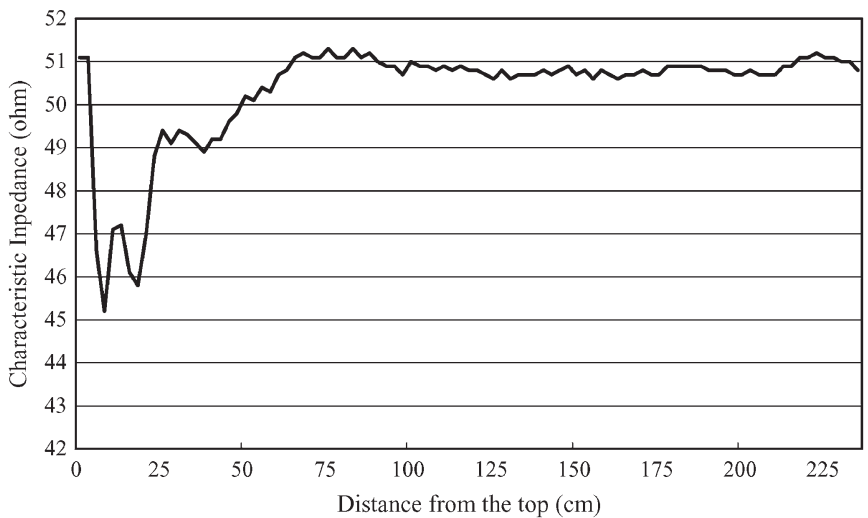

Fig. 7. Measured characteristic impedances along the cell obtained using a TDR.

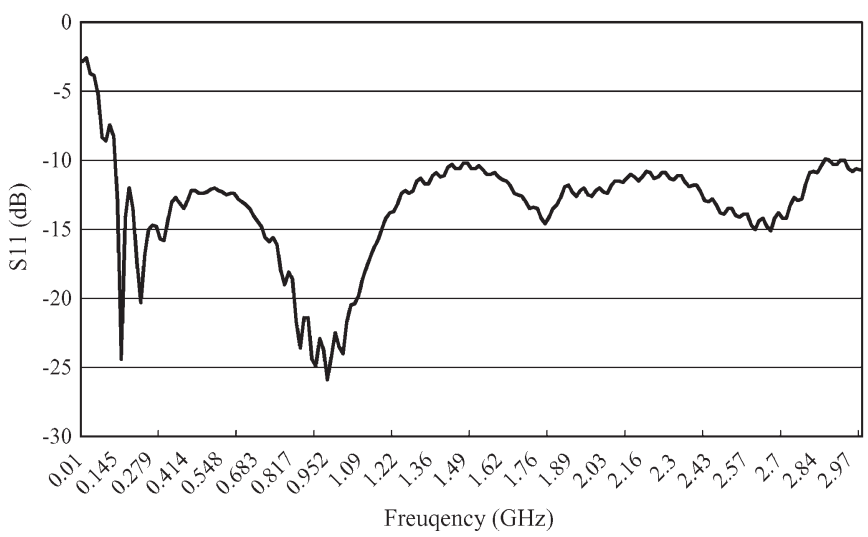

Fig. 8. Measured reflection performance (S11 in decibels) of the tapered cell.

to $2.8 \mathrm{GHz}$. However, our requirement is from $200 \mathrm{MHz}$ to $2.5 \mathrm{GHz}$. Therefore, only this frequency band is used and evaluated. The causes of reflections were also checked using the VNA with a time-domain function. The results indicate that the main reflection comes from the adaptor. The reflection from the bottom (absorbers) is relatively small.

\section{B. Field Distribution}

Field distribution and uniformity are affected by the cell structure and the performance of the absorbers. The absorbers are difficult to accurately characterize in practice. Consequently, simulation and theoretical prediction may be inaccurate. Therefore, this study performed direct E-field measurements.

A calibrated E-field probe was used in the E-field measurements. The field strength measurement error caused by probe linearity is less than $1 \%$. The probe consists of two orthogonal dipole sensors, each fabricated on an opposite side of a small PCB. Each dipole sensor is composed of a short dipole antenna (1 cm in length), a diode detector at the dipole feed-point, and a high-resistance line. A 3-D mechanical scanner was used to perform automatic measurement. The probe was supported by a dielectric rod during measurement.

Three areas were scanned, as shown in Fig. 9. Area 1 and Area 2 are for observing the field distributions, and Area 3 is for calculation of uniformity. The measurement point spacing was $1 \mathrm{~cm}$ for $x$ - and $y$-directions. Area 1 and Area 2 had dimensions of $28 \mathrm{~cm} \times 15 \mathrm{~cm}$. The scanning area was divided into two sections because the maximum traveling distance of our scanner was $28 \mathrm{~cm}$. The size of Area 3 was $10 \mathrm{~cm} \times 10 \mathrm{~cm}$. Two 


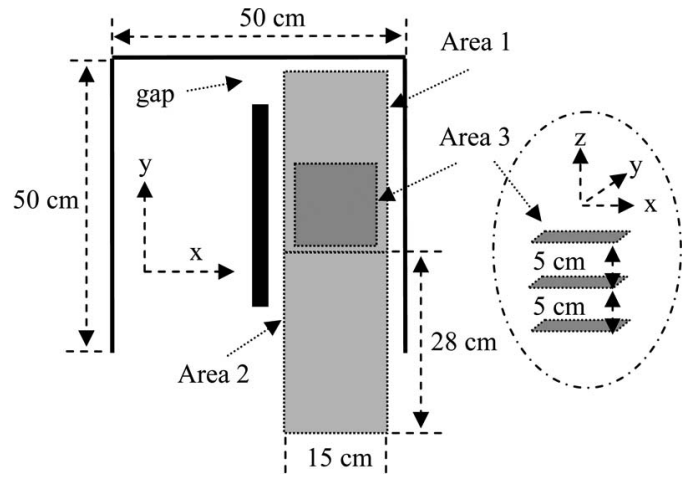

Fig. 9. Selected areas for field distribution and uniformity measurement.

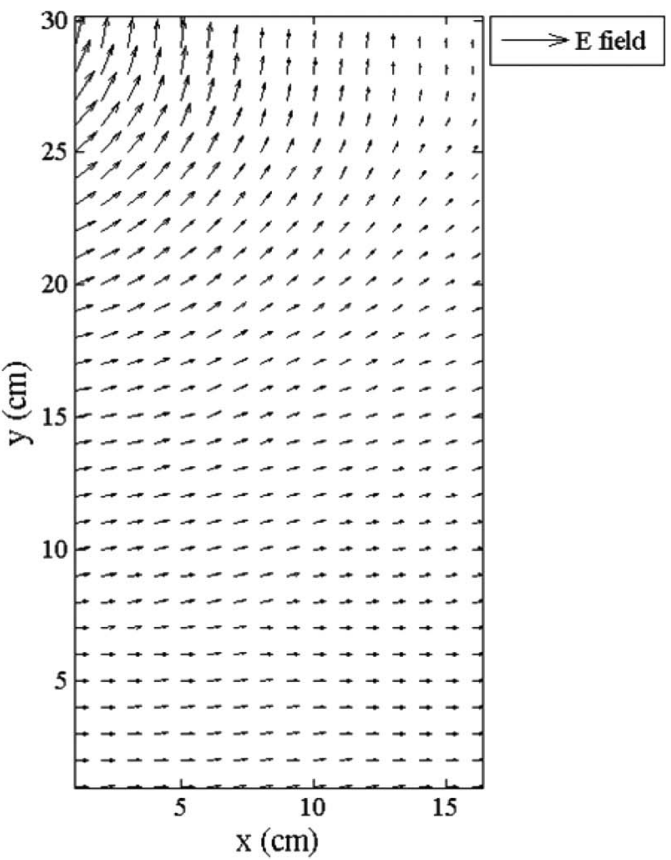

(a)

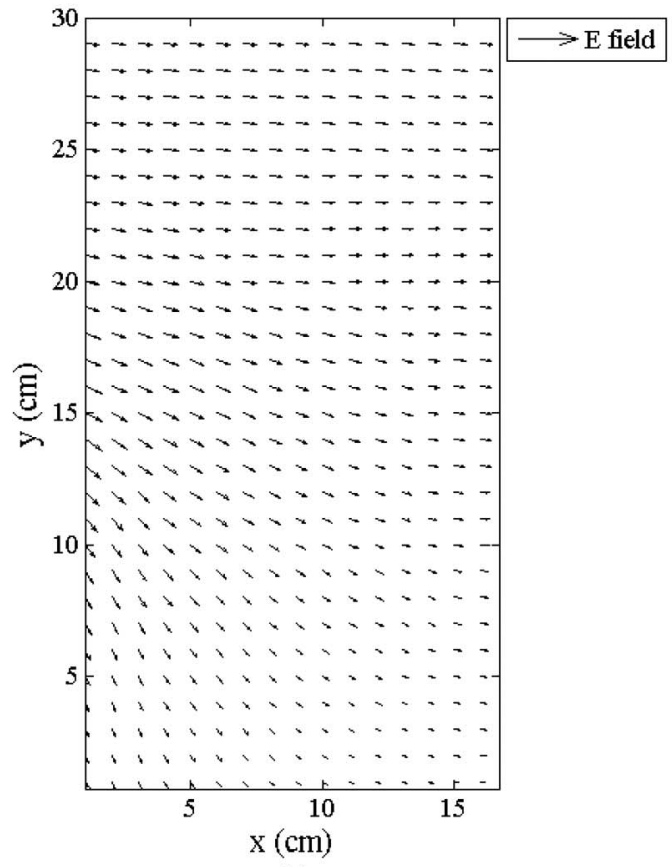

(b)

Fig. 10. Measured field distribution in (a) Area 1 and in (b) Area 2.

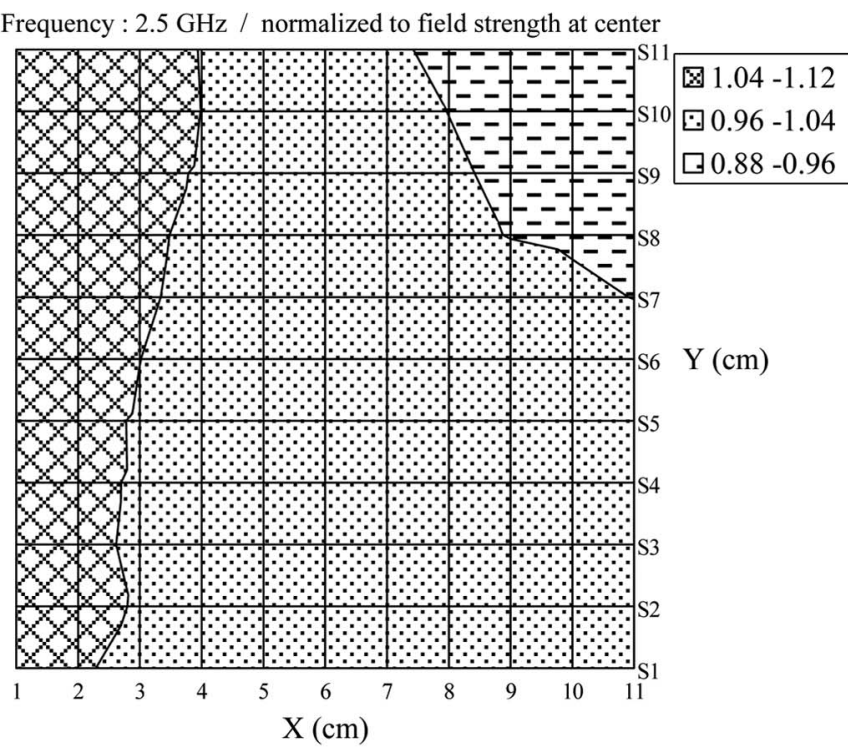

(a)

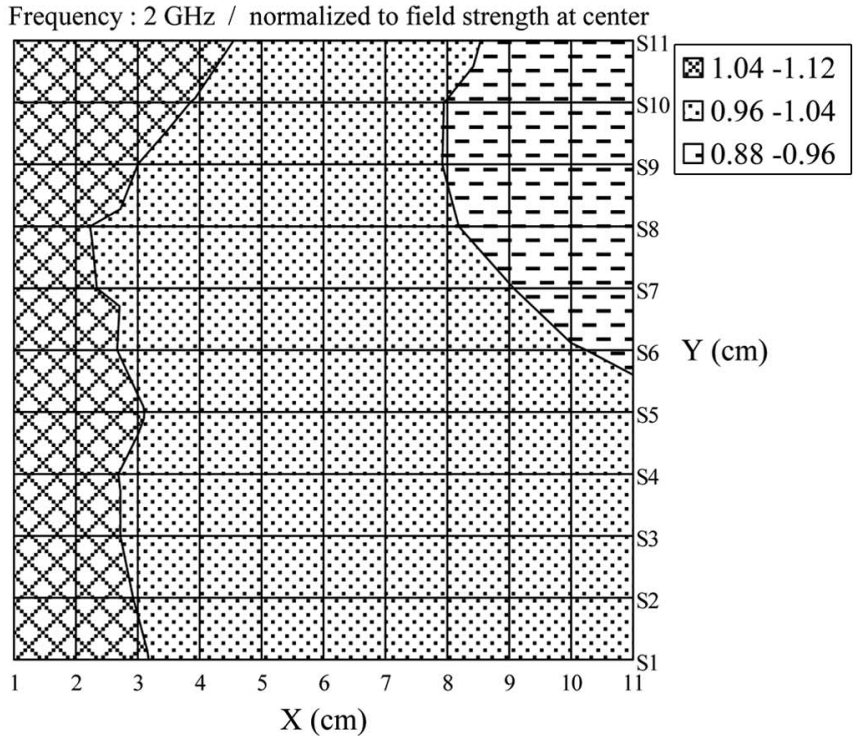

(b)

Fig. 11. Field uniformity of a selected test area between the center septum and the sidewall (Area 3). (a) At $2.5 \mathrm{GHz}$. (b) At $2 \mathrm{GHz}$.

additional areas of the same dimensions under Area 3, which was spaced by $5 \mathrm{~cm}$, were also scanned, as shown in Fig. 9. The measured field strengths of the three scanned squares were used to evaluate the uniformity of a $10 \mathrm{~cm} \times 10 \mathrm{~cm} \times 10 \mathrm{~cm}$ cube.

Fig. 10 shows the scanned field distributions (vectors) at $2.5 \mathrm{GHz}$. The E-field strength was $30 \mathrm{~V} / \mathrm{m}$ at the center of the cell (same as the center of Area 3). In Area 1, the E-field is nearly horizontally polarized near the center of the cell and gradually becomes vertically polarized at positions near the back wall. The strongest field strength is observed near the edge of the center septum. This is predictable because the gap between the edge and the back wall is relatively small. In Area 2, the E-field is also horizontally polarized near the center of the cell and gradually changes direction at the open side (fringe field). Combining the field distributions of Area 1 and Area 2, we can conclude that the wave is in TEM mode at $2.5 \mathrm{GHz}$ (the highest operating frequency). 
Uniformity is one important factor in uncertainty evaluation of probe calibrations and typically depends on the frequency. Therefore, in this study, the uniformity of the specified test volume was measured at every $100-\mathrm{MHz}$ interval, beginning at $200 \mathrm{MHz}$ and ending at $2.5 \mathrm{GHz}$. The uniformity is defined as two times the standard deviation of the field strength distribution in the test volume. Fig. 11 illustrates the uniformity in Area 3 at 2.5 and $2 \mathrm{GHz}$. The measured field strength at every measurement point is normalized to the field strength at the center. It is observed that the field is uniform around the center. The strongest field is found at the area closest to the center septum. The calculated field uniformity in the $10 \mathrm{~cm} \times 10 \mathrm{~cm} \times 10 \mathrm{~cm}$ cube is approximately $\pm 11 \%$ and $\pm 10 \%$ at 2.5 and $2 \mathrm{GHz}$, respectively. If a cube of $5 \mathrm{~cm} \times 5 \mathrm{~cm} \times 5 \mathrm{~cm}$ is considered, the uniformity is only $\pm 7 \%$ at both 2.5 and $2 \mathrm{GHz}$. The results of the field distribution and uniformity measurements demonstrate that the cell is useful for the calibration of E-field probes.

\section{CONCLUSION}

This paper has introduced the structure of a tapered cell and proposed a design methodology. Based on the developed design rules, a tapered cell was constructed and evaluated. The results demonstrate that the cell is useful for E-field probe calibrations.

Using tapered cells is a convenient method to generate EM fields. The application of tapered cells may be extended to EMC tests or studies on the biological effects of E-fields. This study is useful for the construction of tapered cells and for developing their applications.

\section{REFERENCES}

[1] Electromagnetic compatibility (EMC)—Part 4-3: Testing and Measurement Techniques—Radiated, Radio-Frequency, Electromagnetic Field Immunity Test, IEC 61000-4-3, 2006.

[2] IEEE Recommended Practice for Measurements and Computations of Radio Frequency Electromagnetic Fields With Respect to Human Exposure to Such Fields, $100 \mathrm{kHz}-300 \mathrm{GHz}$, IEEE Std. C95.3, 2002.

[3] IEEE Standard for Calibration of Electromagnetic Field Sensors and Probes, Excluding Antennas, From $9 \mathrm{kHz}$ to $40 \mathrm{GHz}$, IEEE Std. 1309, 1996.

[4] M. L. Crawford, "Generation of standard EM fields using TEM transmission cells," IEEE Trans. Electromagn. Compat., vol. EMC-16, no. 4, pp. 189-195, Nov. 1974.

[5] N. S. Nahman, M. Kanda, E. B. Larsen, and M. Crawford, "Methodology for standard electromagnetic field measurements," IEEE Trans. Instrum. Meas., vol. IM-34, no. 4, pp. 490-503, Dec. 1985.

[6] J. Glimm, K. Munter, R. Pape, M. Spitzer, and T. Schrader, "Traceability and uncertainty of field-strength measurements," in Proc. CPEM Conf. Dig., Jul. 1998, pp. 636-637.

[7] W. T. Shay, W. P. Hong, and R. R. Lao, "Calibrating E-field probes with a tapered cell," in Proc. CPEM Conf. Dig., Jun. 2010, pp. 553-554.

[8] J. P. M. de Vreede, G. M. Teunisse, K. Miinter, H. Hoitink, K. Holland, M. O'Dwyer, L. Bergsten, and L. Puranen, "Calibration of RF field strength meters," IEEE Trans. Instrum. Meas., vol. 44, no. 2, pp. 351354, Apr. 1995

[9] D. M. Pozar, Microwave Engineering. Hoboken, NJ, USA: Wiley, 2005, p. 52.

[10] T. S. Chen, "Determination of the capacitance, inductance, and characteristic impedance of rectangular lines," IRE Trans. Microw. Theory Tech., vol. 8, no. 5, pp. 510-519, Sep. 1960.

[11] W. T. Shay, W. P. Hong, and R. R. Lao, "Design and construction of tapered cells," in Proc. CPEM Conf. Dig., Jul. 2012, pp. 646-647.

[12] S. B. Cohn, "Problems in strip transmission lines," IRE Trans. Microw. Theory Tech., vol. 3, no. 2, pp. 119-126, Mar. 1955.

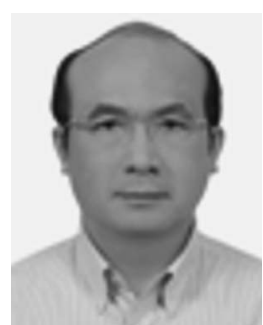

Wen-Tron Shay (M'95-A'96-M'97) received the B.S. degree in electrophysics from National Chiao Tung University, Hsinchu, Taiwan, in 1987. He is currently working toward the Ph.D. degree in communication engineering.

Since 1989, he has been with the Electrical and Electromagnetic Measurement Laboratory, Center for Measurement Standards, Industrial Technology Research Institute, Hsinchu. His current research interests include electromagnetic field metrology, antenna for body area networks, and techniques for measuring and monitoring electromagnetic radiation from modern wireless communication systems

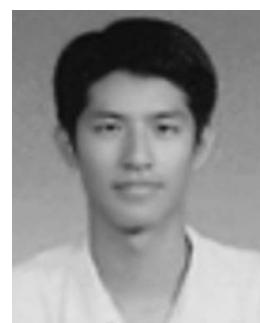

processing

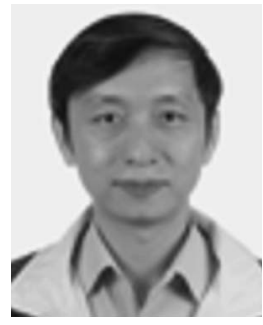

Ray-Rong Lao received the B.Sc. degree in electronic engineering from National Taiwan Ocean University, Keelung, Taiwan, in 1986 and the M.Sc. degree in electrical engineering from Tatung University, Taipei, Taiwan, in 1988.

Since 1990, he has been with the Center for Measurement Standards, Industrial Technology Research Institute, Hsinchu, Taiwan, where he is currently a Principal Researcher in the radio-frequency area and the Manager of the Electrical and Electromagnetic Measurement Laboratory. His main areas of expertise include electromagnetic field and antenna metrology.

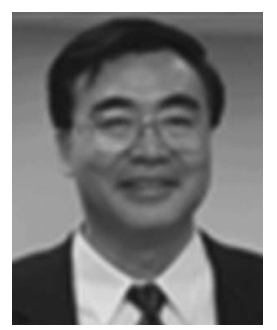

Jenn-Hwan Tarng (S'85-M'86-SM'06) received the $\mathrm{Ph} . \mathrm{D}$. degree in electrical engineering from Pennsylvania State University, University Park, PA, USA, in 1989.

After obtaining his Ph.D. degree, he joined the Faculty of National Chiao Tung University (NCTU), Hsinchu, Taiwan, where he is currently a Professor with the Department of Electrical Engineering. During 2003-2005, he was the Chairman of the Communication Engineering Department and the Director of the Advanced Radio Technology and Systems Center, NCTU. From 2005-2007, he was invited (on leave) as a Chair Professor and Dean of the College of Engineering, Chung Hua University, Hsinchu. Since October 2007, he has been on leave from the Industrial Technology Research Institute, Hsinchu, to be the General Director of the Identification and Security Technology Center. He has published more than 50 refereed journal papers, 60 conference papers, and three book chapters. He is the holder of one U.S. patent and has four U.S. patent applications pending. His professional interests include medical body area network, radio-frequency identification/internet of things, radio channel modeling and measurement, and antenna and radio frequency integrated circuit design.

Dr. Tarng is the Advance Program Chair and Technical Program Committee Member and Cochair of Antennas and Propagation track for the IEEE 71st Vehicular Technology Conference (VTC 2010-Spring), Taipei, Taiwan, May 16-19, 2010. He has also served as a Member of the Technical Planning Committee of several IEEE-sponsored technical conferences, including the IEEE International Conference on Networking, Sensing and Control and the IEEE International Conference on Systems, Man and Cybernetics. He has led and co-led several National Academic Research Programs, such as "Program for Promoting Academic Excellence of Universities-Phases I and II" supported by the Ministry of Education and the National Science Council of Taiwan. 